\title{
Sampling Bloom Filter-Based Detection of Unknown RFID Tags
}

\author{
Xiulong Liu, Heng Qi, Keqiu Li, Senior Member, IEEE, Ivan Stojmenovic, Fellow, IEEE, \\ Alex X. Liu, Yanming Shen, Wenyu Qu, and Weilian Xue
}

\begin{abstract}
Unknown RFID tags appear when the unread tagged objects are moved in or tagged objects are misplaced. This paper studies the practically important problem of unknown tag detection while taking both time-efficiency and energy-efficiency of battery-powered active tags into consideration. We first propose a Sampling Bloom Filter which generalizes the standard Bloom Filter. Using the new filtering technique, we propose the Sampling Bloom Filter-based Unknown tag Detection Protocol (SBF-UDP), whose detection accuracy is tunable by the end users. We present the theoretical analysis to minimize the time and energy costs. SBF-UDP can be tuned to either the time-saving mode or the energy-saving mode, according to the specific requirements. Extensive simulations are conducted to evaluate the performance of the proposed protocol. The experimental results show that SBF-UDP considerably outperforms the previous related protocols in terms of both time-efficiency and energy-efficiency. For example, when 3 or more unknown tags appear in the RFID system with 30000 known tags, the proposed SBF-UDP is able to successfully report the existence of unknown tags with a confidence more than 99\%. While our protocol runs 9 times faster than the fastest existing scheme and reducing the energy consumption by more than $80 \%$.
\end{abstract}

Index Terms-RFID, unknown tags detection, energy-efficiency, time-efficiency.

Manuscript received March 22, 2014; revised August 9, 2014 and December 14, 2014; accepted January 28, 2015. Date of publication February 11, 2015; date of current version April 14, 2015. This work is supported by the National Science Foundation for Distinguished Young Scholars of China (Grant No. 61225010); the State Key Program of National Natural Science of China (Grant No. 61432002); NSFC under Grant nos. of 61173161, 61173162, 61272417, 61300187, 61300189, 61370198, 61370199, 61472184, 61321491, 61272546, and 61173160; the Jiangsu Future Internet Program under Grant No. BY2013095-4-08, and the Jiangsu High-level Innovation and Entrepreneurship (Shuangchuang) Program. A preliminary version [X. Liu et al., "Time- and energy-efficient detection of unknown tags in large-scale RFID systems," in Proc. IEEE MASS, 2013, pp. 95-103] of the paper has appeared in IEEE MASS 2013. The associate editor coordinating the review of this paper and approving it for publication was $\mathrm{V}$. Wong.

X. Liu, H. Qi, K. Li, and Y. Shen are with the School of Computer Science and Technology, Dalian University of Technology, Dalian 116023, China (e-mail: keqiu@dlut.edu.cn).

I. Stojmenovic is with the SIT, Deakin University, Burwood, VIC 3125 , Australia, with King Abdulaziz University, Jeddah 22254, Saudi Arabia, and also with the School of Electrical Engineering and Computer Science (SEECS), University of Ottawa, Ottawa, ON K1N 6N5, Canada (e-mail: stojmenovic@gmail.com).

A. X. Liu is with the Department of Computer Science and Engineering, Michigan State University, East Lansing, MI 48824 USA, and also with the National Key Laboratory for Novel Software Technology, Nanjing University, Nanjing 210008, China (e-mail: alexliu@cse.msu.edu).

W. Qu is with the School of Information and Technology, Dalian Maritime University, Dalian 116026, China (e-mail: eunice.qu@gmail.com).

W. Xue is with the School of Management, Liaoning Normal University, Dalian 116026, China (e-mail: xueweilian@lnnu.edu.cn).

Color versions of one or more of the figures in this paper are available online at http://ieeexplore.iee.org.

Digital Object Identifier 10.1109/TCOMM.2015.2402660

\section{INTRODUCTION}

\section{A. Background}

$\mathbf{R}$ ADIO Frequency Identification (RFID) technology is a type of wireless technique that can automatically identify or track the RFID tags attached to objects or even humans. Compared with the conventional bar-code systems, RFID technique owns many attractive advantages, such as remote and multiple access, simple computational ability, and non-sight limitation, etc. Therefore, it is widely used in the the localization [1]-[3], warehouse monitoring [4]-[6] and supply chain management [7]-[10]. A typical RFID system consists of a back-end server, a single (or multiple) reader(s) and a large number of tags [11]. The tags are attached to items, and each one has a small memory to store its unique ID (96-bit) for individual identification and some information (e.g., product price, shelf-life, etc.) of the tagged item. The RFID tags are generally classified into two types: active tags and passive tags. The active RFID tag uses an internal battery to continuously power the RF communication circuitry, whereas, the passive RFID tag relies on RF energy from the reader to power its circuitry.

\section{B. Motivation and Problem Statement}

In most RFID-enabled applications such as inventory control and warehouse management, normally, the ID information of all tags are stored in a database of the backend server. When some tags are moved out, the corresponding ID information are deleted from the database; when new tags are moved in, the corresponding ID information obtained from tag identification protocols [12], [13] are added into database. However, due to errors in the communication link, e.g., caused by the obstacles in the radio path, some new tags may be left unread [14], [15], and thus unknown tags appear. On the other hand, the misplacement of tagged items can also result in the unknown tags because the misplaced tags are "strangers" to the reader locating in this region. The chilled food will quickly decay if it is misplaced at the zone with no refrigeration equipment and not discovered in time. More seriously, in the warehouse management, a tagged lighter that is misplaced in the area of alcohol may cause fatal safety problem [16]. For reader, both the unread tags and the misplaced tags are unknown tags.

The Unknown Tag Problem (UTP) can be generally classified into two sub-problems: detection and identification. The 
unknown tag detection problem concentrates on discovering the existence of the unknown tags. In contrary, the unknown tag identification problem aims at pinpointing the exact unknown tags. Intuitively, the identification process consumes more time (and energy when active tags are used) than detection process, because identification needs to reports all information about unknown tags while detection only needs to reports the existence of unknown tags (i.e., YES or NO). The heavy identification process sometimes does not discover any unknown tags and thus wastes a lot of time because there are no unknown tag at all. Inspired by [17], a wise way is to periodically perform the tiny unknown detection protocol, and the identification protocol (e.g., BUIP-CF) is invoked only when the detection protocol reports the existence of unknown tags.

In this paper, we study the problem of unknown tag detection, which is formally defined as follows. We target at providing a detection protocol to discover the existence of unknown tags with a reliability of at least $\beta$ if $\lambda$ or more unknown tags appear in the RFID system that consists of $N$ known tags.

\section{Existing Work and Limitations}

The Single Echo based Batch Authentication (SEBA) protocol proposed in [18] uses the well-known Framed Slotted Aloha communication mechanism to detect the existence of unknown tags (called counterfeit tags in [18]). The unexpected response received by the reader is treated as the evidence of unknown tags. SEBA+ [19] exploits the standard Bloom Filter (BF) technique to generalize the SEBA. In SEBA+, each tag randomly selects $r \geq 1$ slots to respond within a frame. As long as one responding location of a unknown tag is not covered by response(s) from known tags (called legitimate tags in [18]), the existence of unknown tags is discovered. SEBA is a special case $(r=1)$ of SEBA+.

These two protocols have three drawbacks: (1) The Bloom Filter is constructed distributively by tags on the slot level. According to Philips I-Code specification [20], any two consecutive transmissions (from a tag to a reader or vice versa) are separated by a waiting time $t_{0}=302 u s$. Hence, constructing $\mathrm{BF}_{T \Rightarrow R}$ (from tags to reader) is time-consuming. For example, if we want to construct a BF with 1000 bits, the time cost of $\mathrm{BF}_{T \Rightarrow R}$ is $1000 \times\left(t_{0}+t_{b i t}\right)$, where $t_{b i t}$ is the time of transmitting 1-bit information from a tag to the reader. It involves a large amount of waiting time, which leads to the low timeefficiency. (2) SEBA and SEBA+ leverage the expected empty slots to detect the unknown tags. To guarantee the required detection accuracy, the ratio of expected empty slots to the whole frame size should be kept at a certain level. Therefore, the frame size is necessary to be proportional to the tag population size, which leads to the poor scalability. (3) Energy-efficiency, an important metric when active tags are used, is not considered in [18], [20].

\section{Main Contributions}

According to the investigation of the prior work, we think the problem of unknown tag detection is of practical importance and still soliciting new efficient solutions. To this end, this paper studies the problem of unknown tag detection and makes the following contributions:

1) We combine the standard Bloom Filter and sampling idea to propose the Sampling Bloom Filter (SBF), in which no element is necessary to use $k$ times hashing, however, each of the $k$ hashings depends on the the sampling probability.

2) We exploit the new filtering technique to propose the Sampling Bloom Filter-based Unknown tag Detection Protocol (SBF-UDP), in which the reader constructs a filter according to the IDs of known tags. Then, the filter is broadcasted to all tags for verifying their membership.

3) This paper takes both time-efficiency and energyefficiency into account. Sufficient theoretically analysis are proposed to investigate the parameter settings to minimize the time cost (or energy consumption) while guaranteeing the predefined detection reliability.

4) Extensive simulations are conducted to evaluate the performance of the proposed protocol. The experimental results demonstrate that the proposed protocol considerably outperforms the existing related protocols in terms of both time-efficiency and energy-efficiency.

The rest of this paper is organized as follows. The related work is reviewed in Section II. Section III describes the preliminary knowledge including system model and the used performance metrics. We propose the SBF-UDP as well as the theoretical analyzes of the parameter settings in Section IV. In Section V, extensive simulation experiments are conducted to evaluate the performance of the proposed SBF-UDP. This paper is concluded in Section VI.

\section{RELATED WORK}

RFID is an emerging technology that is widely used in many monitoring applications. There are two basic monitoring tasks that solicit efficient solutions: missing tag problem and unknown tag problem.

Missing Tag Detection: In recent years, many efforts have been made to address the missing tag problem. Tan et al. proposed the Trust Reader Protocol (TRP) to detect the missingtag event with a predefined probability $\beta$ when the number of the missing tags exceeds a given threshold [21], which also inspired the problem formulation of this paper. To improve the time-efficiency and energy-efficiency of TRP, Luo et al. introduced the sampling idea, and thus proposed the Efficient Missing-tag Detection (EMD) protocol, where they used the detection result on the sampled tags to probabilistically reflect the whole intactness [22]. However, EMD still has a large room to be improved because it contains a large proportion of expected empty/collision slots that cannot be used in missing tag detection. To overcome this deficiency, Luo et al. studied a multi-hashing-seed approach to reduce the useless empty slots and collision slots involved in the EMD protocol and thus proposed the Multi-Seed Missing-tag Detection (MSMD) protocol [17]. These protocols (TRP [21], EMD [22] and MSMD [17]) concentrate on discovering the missing-tag event, instead of pinpointing which tags are missing. 


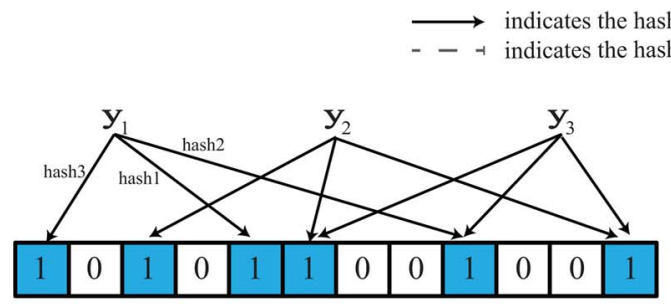

(a)

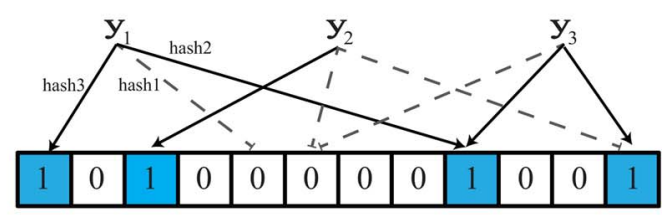

(b)

Fig. 1. Standard Bloom Filter vs. Sampling Bloom Filter. (a) Standard Bloom Filter: each element is pseudo-randomly compressed into the filer using $k$ different hashing functions. (b) Sampling Bloom Filter: each element is also pseudo-randomly mapped to the filter with $k$ hashing functions, however, each mapping is implemented with a sampling probability $p$, and the sampling process is also pseudo-random.

Missing Tag Identification: The Iterative ID-free Protocol (IIP) proposed in [23] is a variant of the Framed Slotted Aloha protocol, and is able to pinpoint the exact missing tags. The protocols proposed by Zhang et al. in [24] accelerate the protocol's execution by leveraging the collaboration of multiple readers. To improve the slot utilization, an efficient collision reconciling method was proposed to change some collision slots into singleton slots that can be used for the identification of missing tags [25]. Zheng et al. investigated the compressive sensing technique to perform the identification at the physicallayer [26].

Unknown Tag Identification: The unknown tag problem is also very important in practice [27]. As aforementioned, the unknown tagged objects such as misplaced or left unread items could cause serious economic loss or security issues. The existing protocols dedicated for unknown tag detection have been reviewed in Section I. In terms of unknown tag identification, Sheng et al. exploited the Framed Slotted Aloha mechanism to propose a CU (Collect Unknown tags) [28]. The tags responding the expected empty slots are necessary to be unknown tags, and will keep active to be collected by Enhanced Dynamic Framed Slotted ALOHA (EDFSA) protocol [29]. Multiple rounds are required to achieve desired identification accuracy. In the $\mathrm{CU}$ protocol, only the expected empty slots are used to detect the unknown tags. However, other slots are not fully explored. Moreover, all known tags participate in each round, which interferes the unknown tag detection. To overcome these drawbacks of the CU protocol, the BUIP-CF protocol proposed in [30] uses, in addition to the expected empty slots to label the unknown tags, also the expected singleton slots to deactivate the known tags (preventing them from interfering the detection of unknown tags). Specifically, if one and only one tag responds in an expected singleton slot, this must be known tag. Then, the reader sends an ACK signal to deactivate it (i.e., to enter the sleep state). However, if one or more tags respond in an expected empty slot, all of them must be unknown tags. Then, the reader sends a NACK signal to label them (i.e., telling them not to participate in the next round, but still remain active). In a round, some known/unknown tags could be deactivated/labeled, and they will not participate in the following rounds; the other tags will participate in the next round. This process is repeated for multiple rounds until all known tags are deactivated. In contrary, all the unknown tags keep active and will be collected by the EDFSA protocol [29]. Liu et al. proposed to use bit vector (from reader to tags) to fast filter out the unknown tags [31] for the purpose of unknown tag identification. Further, they investigated a bitwise XOR filter technique, the " $1 \mathrm{~s}$ " in the XOR filter can not only label the unknown tags but al accelerate the unknown ID collection [27].

Bloom Filter Technique: A Bloom Filter [32]-[34] is a wellknown data structure that is very popular in database applications and also receives widespread attention in the networking literature. Bloom Filter $(\mathrm{BF})$ probabilistically represents a set of $n$ elements $Y=\left\{y_{1}, y_{2}, \cdots, y_{n}\right\}$, which can be used to test set membership. Specifically, the Bloom Filter compresses this set into a filter vector with $w$ bits by hashing each element in $Y$ into the vector using $k$ hashing functions $H_{1}, H_{2}, \cdots, H_{k}$. As illustrated in Fig. 1(a), a bit in the vector is set to " 1 " if at least one element is hashed to that index in the vector; otherwise, it is set to " 0 ". When checking whether a given element $y$ belongs to the set $Y$, we compute $H_{1}(y), H_{2}(y), \cdots, H_{k}(y)$ and assert $y \in S$ if and only if all these $k$ bits are " $1 \mathrm{~s}$ " in the vector; otherwise, $y \notin Y$. Bloom filter technique is relatively lightweight and has potential to be used on RFID devices. For example, [4], [8] use Bloom filter or its variant to search the exact wanted tags in a given RFID system.

\section{PRELIMINARY}

\section{A. System Model and Assumption}

We assume a large-scale RFID system that consists of a backend server, a reader, $N$ known tags, and $M$ unknown tags, where usually $M \ll N$. Let $T_{\Delta}$ denote the known tag set, i.e., $T_{\Delta}=\left\{t_{1}, t_{2}, \ldots, t_{i}, \ldots, t_{N}\right\}$, and the number $N$ as well as ID information in $T_{\Delta}$ is available in a database on the backend server. The unknown tag set is denoted as $T_{\Lambda}$, i.e., $T_{\Lambda}=$ $\left\{t u_{1}, t u_{2}, \ldots, t u_{i}, \ldots, t u_{M}\right\}$, whereas, both the number $M$ and the specific ID information in $T_{\Lambda}$ are not known in advance. Each tag has a unique ID and is equipped with the same uniform hash generator $H(\cdot)$. We assume the reader has adequate power to interrogate all the tags including the known ones and the unknown ones. Moreover, the reader communicates with the back-end server via a high-rate network link, and has access to the ID information of all known tags.

The reader communicates with the tags in a time-slotted way, where the slots are synchronized by the periodical "end slot" commands broadcasted by the reader. According to the specification of the Philips I-Code system [20], the wireless transmission rate from a tag to a reader is $53 \mathrm{~Kb} / \mathrm{s}$ and the rate from a 
reader to a tag is $26.5 \mathrm{~Kb} / \mathrm{s}$. Any two consecutive transmissions (from a tag to a reader or vice versa) are separated by a waiting time $t_{0}$ of 302 us [16]. The duration of a slot that supports transmission of $\mu$-bit data is $t_{0}+\mu t_{b i t}$, where $t_{b i t}$ is the time for transmitting 1 bit. Usually, $\mu$ is less than 96, i.e., the length of tag ID. Following prior literature [23], the slots are classified into tag slots, long-response slots and short-response slots. The length of a tag slot is denoted as $t_{t a g}$, which allows the transmission of a tag ID (96 bits), either from the reader to the tags or from a tag to the reader. The length of a long-response slot is denoted as $t_{\text {long }}$, and is sufficient to transmit 10 bits information. The length of a short-response slot is denoted as $t_{\text {short }}$, and allows the transmission of only 1 bit information. In this paper, $t_{t a g}$ is set to be $4 \mathrm{~ms}$ for transmission of a tag ID (96 bits) from a tag to a reader or vice versa. Similarly, $t_{\text {long }}$ and $t_{\text {short }}$ are set to be $0.7 \mathrm{~ms}$ and $0.4 \mathrm{~ms}$, respectively.

\section{B. Energy Consumption Model}

Because the battery of a reader can be easily recharged or the reader may even use an external power source [17], the energy consumed by the reader is ignored in this paper. Accordingly, we only consider the energy consumption of the batterypowered active tags, particularly, the known tags. During a slot, an active tag has two types of states: awake state and sleep state [35]. Specifically, a tag needs to be in the awake state (i.e., its CPU works at full energy and the radio remains active) for communication. An awake tag may operate one of the three actions during a certain slot: transmitting data to the reader; receiving data from the reader; or just listening the channel for the periodical 'end-slot' commands broadcasted by the reader. Since the radio scanning consumes most of the energy, the above actions of an awake tag almost consume the same amount of energy. Similar assumptions are also made in [36]. Let $\omega$ denote the energy consumption of an awake tag during a tag slot. Since the length of a short-response slot is $\frac{1}{10}$ of the tag slot, the energy consumption of an awake tag during the short-response slot is about $\frac{1}{10} \omega$. For similar reason, the energy consumed in a long-response slot is about $\frac{7}{40} \omega$. To conserve battery power, the tag can enter the sleep state, where the CPU works in a low power mode and radio reception is disabled. The ratio of energy consumed between the awake and sleep states is typically on the order of 100 or more [35], so we neglect the energy consumption of an asleep tag. We use $E$ to denote the energy consumption of $N$ known tags, which is given as $E=$ $\sum_{i=1}^{N}\left[\eta_{i 1} \cdot\left(\frac{1}{10} \omega\right)+\eta_{i 2} \cdot\left(\frac{7}{40} \omega\right)+\eta_{i 3} \cdot \omega\right]$, where $\eta_{i 1}, \eta_{i 2}$ and $\eta_{i 3}$ indicate the number of the short-response slots, the longresponse slots and the tag slots that tag $t_{i}$ keeps awake for, respectively. The used notations are summarized in Table I.

\section{Performance Metrics}

We consider two important performance metrics: (1) execution time: we obviously desire to discover the unknown tag event as soon as possible, and thus we are able to take timely countermeasures, e.g., replacing the chilled food to the zone equipped with freezers. (2) energy consumption: the battery volume of active tag is typically limited and replacing batteries
TABLE I

NOTATIONS USED IN THIS PAPER

\begin{tabular}{|c|l|}
\hline Symbols & Descriptions \\
\hline$I D$ & ID information of a RFID tag \\
\hline$N$ & number of known tags in the system \\
\hline$M$ & number of unknown tags in the system \\
\hline$\lambda$ & tolerance threshold \\
\hline$\beta$ & detection reliability \\
\hline$T_{\Delta}$ & set of all known tags in the system \\
\hline$T_{\Lambda}$ & set of all unknown tags in the system \\
\hline$t_{t a g}$ & length of a tag slot \\
\hline$t_{l o n g}$ & length of a long-response slot \\
\hline$t_{s h o r t}$ & length of a short-response slot \\
\hline$\omega$ & energy consumption of an awake tag during a tag slot \\
\hline$H(\cdot)$ & hash generator that follows a uniform random distribution \\
\hline$X$ & a large constant pre-configured in the RFID tags \\
\hline$k$ & number of employed hashing functions \\
\hline$p$ & sampling probability \\
\hline$\left\langle p_{t}, k_{t}\right\rangle$ & optimal pair that minimizes the time cost of SBF-UDP \\
\hline$\left\langle p_{e}, k_{e}\right\rangle$ & optimal pair that minimizes the energy cost of SBF-UDP \\
\hline
\end{tabular}

for a large number of tags is quite laborious. Therefore, it is desirable to reduce the energy consumption as much as possible. The protocols proposed in this paper are not limited to the active tags. If only passive tags are used in an application, the time-efficiency becomes the only performance metric.

\section{A Sampling Bloom Filter-BASed UnKNown TAg Detection Protocol}

In this section, we first propose the Sampling Bloom Filter which generalizes the standard Bloom Filter. Then, we present the detailed design of the Sampling Bloom Filter-based Unknown tag Detection Protocol (SBF-UDP). After that, we theoretically investigate how to configure the system parameters, e.g., the number of hashing functions, the sampling probability, the filter length, to guarantee the predefined detection reliability. Finally, we analyze the time cost and energy cost of our SBF-UDP, respectively.

\section{A. Overview of the Sampling Bloom Filter}

Given a set $Y=\left\{y_{1}, y_{2}, \cdots, y_{n}\right\}$ containing $n$ different elements, the principle of the Sampling Bloom Filter is presented as follows. As illustrated in Fig. 1(b), each element is mapped to the filter using $k$ hashing functions. However, different from the standard Bloom Filter, when "inserting" a certain element into the filter, each of the $k$ mappings is really implemented with a sampling probability $p$. For example, the element $y_{1}$ is hashed to the 5th bit using $H_{1}(\cdot)$, but this mapping is not sampled, then the 5th bit will not be set to " 1 ". When checking whether a given element $y$ belongs to $Y$, as long as one of the $k$ mappings is sampled and the corresponding bit is " 0 ", we can assert that $y \notin Y$; otherwise, $y \in Y$. Clearly, the proposed Sampling Bloom Filter is a general version of Bloom Filter. In other words, the standard Bloom Filter is a special case $(p=1)$ of the proposed Sampling Bloom Filter.

\section{B. Protocol Design of SBF-UDP}

The proposed SBF-UDP consists of three stages: (1) Constructing the Sampling Bloom Filter; (2) Verifying the Tag Identity; and (3) Announcing Unknown Identity. 
1) Constructing the Sampling Bloom Filter: The reader has access to a database that stores the ID information of all known tags. The reader then compresses each known tag ID to the $f$-bit filter $F$ using $k$ uniform hashing functions. Specifically, an arbitrary known tag $t_{i}$ with $I D_{i}$ is mapped to the locations $l_{i 1}, l_{i 2}, \ldots, l_{i u}, \ldots, l_{i k}$, where $l_{i u}=H_{u}\left(I D_{i}, R_{1}\right) \bmod f, u \in$ $[1, k]$, and $R_{1}$ is a random seed. The $k$ bits are called the representative bits of tag $t_{i}$. Note that, no representative bit is necessary to be set to " 1 ", which actually depends on the sampling result. Given a sampling probability $p \in(0,1]$, the reader calculates an integer $x=\lceil X \times p\rceil$, where $X$ is a large constant that is pre-configured in the tag chip [22]. The representative bit with location $l_{i u}$ is sampled and set to " 1 " only when $H_{u}\left(I D_{i}, R_{2}\right)$ mod $X \leq x$, where $R_{2}$ is another random number used for sampling process; otherwise, the reader does not change this bit. The Sampling Bloom Filter is completed after all the known IDs are inserted into the filter according to the method described above.

2) Verifying the Tag Identity: Then, the reader broadcasts the employed parameters $\left\langle R_{1}, R_{2}, k, f, x\right\rangle$ along with the constructed $f$-bit filter to all RFID tags. Each tag uses the same $k$ hash functions $H_{1}(\cdot) \sim H_{k}(\cdot)$ and the received parameters to check whether it belongs to the known tag set. Specifically, for an arbitrary tag, it first calculates $H_{u}\left(I D, R_{2}\right) \bmod X$, $u \in[1, k]$. If the hashing result is equal to or less than $x$, it means the $u$ th representative bit of this tag is sampled. Then, the tag further calculates $l_{u}=H_{u}\left(I D, R_{1}\right) \bmod f$. If the $l_{u}$ th bit is " 0 ", this tag will be aware of its unknown identity; otherwise, it will check its other representative bits using the same method. As long as one of the representative bits is sampled but turns out to be " 0 ", the tag is necessary to be unknown tag.

3) Announcing Unknown Identity: At the end of this protocol, the unknown tags that are aware of their unknown identity will respond to the reader to announce its unknown identity. If the reader senses a busy slot carrying announcement(s), it will successfully detect the existence of unknown $\operatorname{tag}(\mathrm{s})$.

Clearly, the actual number of unknown tags has significant impact on the detection results of our SBF-UTD. The more unknown tags there are in the system, the more likely our SBF-UTD detects the existence of unknown tags. (1) If there is no unknown tag, SBF-UTD will not report the existence of unknown tags. (2) If the number of unknown tags is less than the threshold $\lambda$, SBF-UTD can sill have a chance to discover the existence of unknown tags. But we do not guarantee the required reliability of $\beta$. (3) If the number of unknown tags is larger than the threshold $\lambda$, theoretical analysis in the next section can guarantee that the actual reliability of SBF-UTD is larger than $\beta$. No matter how many unknown tags appear in the system, the processes of SBF-UTD is always the same because unknown tags announce their unknown identity at the end of our protocol. Even if there is no unknown tag, SBF-UTD still needs to go through the above three stages. The execution time is independent of the number of unknown tags.

\section{Investigating the Detection Accuracy}

There are two types of false detection for the unknown tag detection problem. (1) The detection protocol reports the existence of unknown tags, although there is no unknown tag (i.e., "false alarm"). (2) The detection protocol does not discover any unknown tags, despite the presence of unknown tags (i.e., "miss-detection"). SBF-UTD does not incur false alarms, because the sampled representative bits of a known tag will always be " $1 \mathrm{~s}$ ". On the other hand, our protocol indeed suffers from the miss-detection, which occurs when all the representative bits of unknown tags are "occupied" by known tags. We formally analyze the miss-detection in this section.

We use $P(N, M, f, k, p)$ to denote the probability of successfully discovering the existence of unknown $\operatorname{tag}(\mathrm{s})$ when there are $N$ known tags and $M$ unknown tags, the filter length is $f$, the sampling probability is $p$, and the $k$ uniform hash functions are used. In the following, we theoretically propose the expression of $P(N, M, f, k, p)$. Let $P_{\text {false }}$ denote the probability that an arbitrary unknown tag eventually passes the detection. A unknown tag can pass the detection only when each of its $k$ representative bits satisfies one of the following conditions: (1) not sampled; (2) if sampled, at least one known tag is hashed to the same location, which sets the representative bit to " 1 ". $P_{\text {false }}$ is expressed as follows:

$$
\begin{aligned}
P_{\text {false }} & =\left\{(1-p)+p\left[1-\left((1-p)+p\left(1-\frac{1}{f}\right)\right)^{N k}\right]\right\}^{k} \\
& =\left(1-p e^{-\frac{N k p}{f}}\right)^{k}
\end{aligned}
$$

As long as one of the $M$ unknown tag is discovered, the existence of unknown tags will be successfully reported. Hence, the detection probability $P(N, M, f, k, p)$ can be given as:

$$
P(N, M, f, k, p)=1-P_{\text {false }}^{M}=1-\left(1-p e^{-\frac{N k p}{f}}\right)^{M k} .
$$

According to (2), we find that $P(N, M, f, k, p)$ monotonically increases as $M$ increases. Hence, when the number $M$ of unknown tags exceeds the threshold $\lambda$, we have $P(N, M, f, k, p) \geq P(N, \lambda, f, k, p)$. Obviously, if we could guarantee $P(N, \lambda, f, k, p) \geq \beta$, the actual detection probability will be larger than $\beta$ for any $M \geq \lambda$. By solving the inequality $P(N, \lambda, f, k, p) \geq \alpha$, we have

$$
f \geq \frac{-N k p}{\ln \left[\frac{1-(1-\beta)^{\frac{1}{\lambda k}}}{p}\right]}
$$

\section{Analyzing the Performance of SBF-UDP}

In this subsection, we analyze the time cost and the energy cost of the proposed SBF-UDP, respectively. Moreover, we investigate how to minimize them.

1) Time Cost: Following [18], [19], [28], [30], we only consider the time consumed by the wireless communication between the reader and the tags, and exclude the time consumed by computation. In the stage of Constructing the Sampling Bloom Filter, one tag slot $t_{t a g}$ is adequate for the reader to 


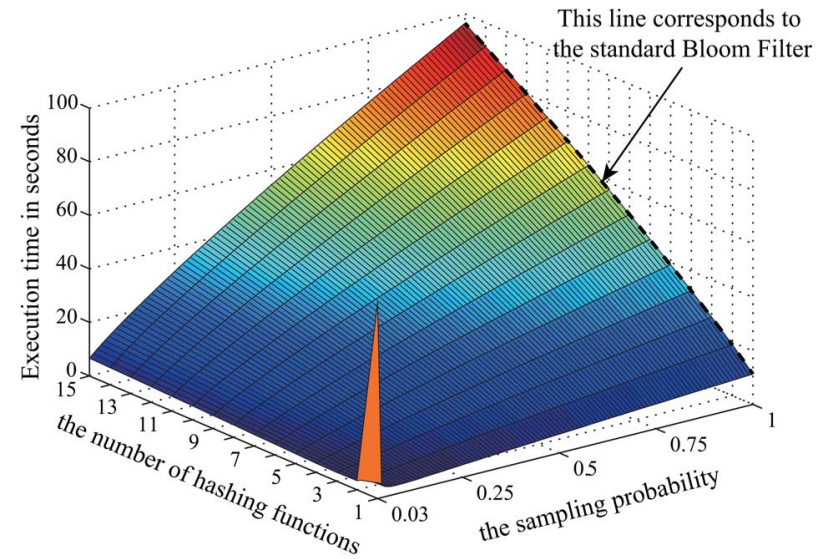

Fig. 2. Investigating the impact of $p$ and $k$ on the time cost of SBF-UDP, where $N=10000, \lambda=100, \beta=95 \%, p$ varies from $3 \%$ to $100 \%$, and $k$ changes from 1 to 15 .

broadcast the initialization parameters. The $f$-bit filter will be divided into $S$ segments of 96-bit to be sequentially transmitted in $S$ tag slots [23] when its length is larger than 96 bits (the maximum number of bits that can be transmitted in a tag slot), where $S=\left\lceil\frac{f}{96}\right\rceil$. Then, the time for by transmitting the filter is $\left\lceil\frac{f}{96}\right\rceil \times t_{\text {tag }}$, where $t_{\text {tag }}$ can afford transmitting 96-bit data.

In the stage of Verifying the Tag Identity, the reader waits one short-response slot $t_{\text {short }}$ for listening the expected announcement from the unknown tags. Hence, the time cost $T$ of SBFUDP is given as follows:

$$
T=t_{\text {tag }}+\left\lceil\frac{f}{96}\right\rceil \times t_{\text {tag }}+t_{\text {short }} .
$$

2) The Minimum Time Cost: According to (3), $f$ should be set larger than $-N k p /\left\{\ln \left[\frac{1-(1-\beta)^{\frac{1}{\lambda k}}}{p}\right]\right\}$ so as to meet the predefined accuracy. Eq. (4) shows that $T$ is an increasing function with respect to $f$. Hence, given $N, \lambda, \beta, k$, and $p$, the filter length $f$ should be minimized to $-N k p /\left\{\ln \left[\frac{1-(1-\beta)^{\frac{1}{\lambda k}}}{p}\right]\right\}$ to achieve the minimum time cost $T_{\min }$. Note that, this is just a local optimization with fixed $p$ and $k$. As illustrated in Fig. 2, different parameter pairs $\langle p, k\rangle$ result in different time cost of SBF-UDP. Note that, the dotted line in Fig. 2 actually corresponds to the use of the standard Bloom Filter (i.e., $p=1$ ), which reveals the superiority of our Sampling Bloom Filter. Then, the next key task is to search the optimal pair $\left\langle p_{t}, k_{t}\right\rangle$ to get the overall minimum time cost of SBF-UDP. Note that, if the sampling probability $p$ is too small, the detection accuracy cannot be larger than the desired reliability $\beta$ for any values of $k$ and $f$. Such a small sampling probability $p$ cannot be used. In what follows, we derive the lower bound $p_{\min }$ of the sampling probability

$$
\begin{aligned}
f \geq \frac{-N k p}{\ln \left[\frac{1-(1-\beta)^{\frac{1}{\lambda k}}}{p}\right]}>0 \\
\Rightarrow p>1-(1-\beta)^{\frac{1}{\lambda k}} .
\end{aligned}
$$

Hence, the lower bound $p_{\min }$ on the sampling probability is $1-$ $(1-\beta)^{\frac{1}{\lambda k}}$. For instance, if the tolerance number $\lambda$ is set to 20 , the number $k$ of used hashing functions is 5 , and the detection reliability $\beta$ is set to $95 \%$, the minimum sampling probability $p_{\min }$ is about $3 \%$.

3) Energy Cost: Recall that the long filter is divided into $S$ segments of 96-bits to be sequentially transmitted, where $S=$ $\left\lceil\frac{f}{96}\right\rceil$. Each tag remains awake before successfully receiving all the segments containing its representative bits. For an arbitrary known tag (we do not consider the energy consumption of the unknown tags), let $L$ be the index of the last segment that contains its representative bit(s). Clearly, this tag has to keep active for $L$ tag slots to receive the first $L$ segments before entering the sleep state. The goal is to calculate the expectation of $L$. This can be treated as a problem of allocating $k$ balls into $S$ bins. The bins are numbered from 1 to $S$. Each of the $k$ balls is thrown with probability $p$ into one of the $S$ bins following uniform distribution. Note that, one bin can contain more than one balls. Question: Which bin is the last one that contains ball(s) on average? We propose Theorem 1 to answer the question.

Theorem 1: Assuming there are $k$ balls to be thrown into $S$ bins numbered from 1 to $S$. For a certain ball, it is determined to throw with a probability $p$. If we determine to throw it, the $S$ bins have the same chance $\frac{1}{S}$ to obtain this ball. After tackling all the balls, the index of the last bin containing ball(s) is denoted as $L$. The expectation $E(L)$ of $L$ is $S-$ $\sum_{i=0}^{S-1}\left(1-p+\frac{i p}{S}\right)^{k}$.

Proof: Let $P[L=u]$ be the probability that the $u$ th bin is the last bin containing at least one ball, where $u \in[0, S] . u=0$ means no balls are thrown. $P[L=u]$ is given as follows:

$$
P[L=u]= \begin{cases}\sum_{i=1}^{k}\left(\begin{array}{c}
k \\
i
\end{array}\right)\left(p \frac{1}{S}\right)^{i}\left[(1-p)+p \frac{u-1}{S}\right]^{k-i} & u \in[2, S] \\
\sum_{i=1}^{k}\left(\begin{array}{c}
k \\
i
\end{array}\right)\left(p \frac{1}{S}\right)^{i}(1-p)^{k-i} & u=1 \\
(1-p)^{k} & u=0 .\end{cases}
$$

According to (6), $P[L=u]$ can be simplified to

$$
P[L=u]= \begin{cases}\left(1-p+\frac{u p}{S}\right)^{k}-\left[1-p+\frac{p(u-1)}{S}\right]^{k} & u \in[1, S] \\ (1-p)^{k} & u=0\end{cases}
$$

The expectation $E(L)$ is given as

$$
E(L)=\sum_{u=0}^{S} u \times P[L=u]=S-\sum_{i=0}^{S-1}\left(1-p+\frac{i p}{S}\right)^{k} .
$$

Corollary 1: For fixed $k$ and $p, E(L)$ is an increasing function with respect to $S$.

Proof: Given a fixed $k, E(L)$ becomes a function with respect to $S$, which is denoted as $Q(S)$. For an arbitrary positive integer $S$, if $Q(S+1)>Q(S)$ always holds, $E(L)$ is proved 
to be an increasing function with respect to $S$. According to (8), $Q(S+1)-Q(S)$ is given as

$$
\begin{aligned}
& Q(S+1)-Q(S) \\
& =\left[(S+1)-\sum_{i=0}^{S}\left(1-p+\frac{i p}{S+1}\right)^{k}\right]-\left[S-\sum_{i=0}^{S-1}\left(1-p+\frac{i p}{S}\right)^{k}\right] \\
& =\underbrace{1-\left(1-\frac{p}{S+1}\right)^{S}}_{>0}-\underbrace{\sum_{i=0}^{S-1}\left[\left(\frac{i p}{S+1}\right)^{k}-\left(\frac{i p}{S}\right)^{k}\right]}_{<0}>0 .
\end{aligned}
$$

Therefore, $Q(S)$ (i.e., $E(L)$ ) is an increasing function with respect to $S$.

Recall the problem of allocating $k$ balls to $S$ bins. The last bin containing ball(s) is expected to be the $E(L)$ th bin, determined by (8). Known tags have to keep awake before receiving the last segment $(E(L)$ th segment) containing its representative bits. Overall, they have to remain awake for one tag slot to receive the initialized parameters and $E(L)=S-$ $\sum_{i=0}^{S-1}\left(1-p+\frac{i p}{S}\right)^{k}$ (expectation value) more tag slots before entering the sleep state. The expected energy consumption of a known tag during one execution of SBF-UDP is $[1+E(L)] \times$ $\omega$. For $N$ known tags in total, the energy cost $E$ of SBF-UDP (excluding the energy consumption of unknown tags) is:

$$
\begin{aligned}
E & =N \times[1+E(L)] \times \omega \\
& =N \times\left[1+S-\sum_{i=0}^{S-1}\left(1-p+\frac{i p}{S}\right)^{k}\right] \times \omega .
\end{aligned}
$$

4) The Minimum Energy Cost: When the battery powered active tags are used, as aforementioned, we may need to minimize the energy cost, thereby prolonging the lifetime of the RFID system. Obviously, the energy cost $E$ of SBF-UDP is an increasing function with respect to $E(L)$. Moreover, according to Corollary 1, $E(L)$ is also an increasing function with respect to $S$. Hence, the energy cost $E$ is an increasing function with respect to $S$, where $S=\left[\frac{f}{96}\right]$. Similar to the analysis of the minimum time cost $T$ in Section IV-D2, the filter length $f$ should be minimized to $-N k p /\left\{\ln \left[\frac{1-(1-\beta) \frac{1}{\lambda k}}{p}\right]\right\}$, thereby achieving the minimum energy cost while meeting the predefined detection accuracy. Note that, this is also a local optimization with given $k$ and $p$. As illustrated in Fig. 3, the parameters $\langle p, k\rangle$ significantly affect the energy cost of SBF-UTP. Again, to get the overall minimum energy cost $E$, searching the optimal parameter pair $p_{e}$ and $k_{e}$ is necessary.

In this paper, we propose a simple Algorithm 1 to find the optimal parameter pair $\left\langle p_{t}, k_{t}\right\rangle$ to achieve the overall minimum execution time and $\left\langle p_{e}, k_{e}\right\rangle$ to achieve the overall minimum energy cost. Specifically, steps $1 \sim 4$ in Algorithm 1 initialize variables. $\delta$ specifies the maximum deviation between the calculated $p$ and its real optimal value. $k$ is typically less than 15 [37]. Therefore, we traverse all values of $k$ between 1 and 15 in step 5. For each $k$, steps 6 23 in Algorithm 1 test $p$ values at $\delta$ intervals apart to find the minimum time cost as well as energy cost, meanwhile recording the corresponding $p$

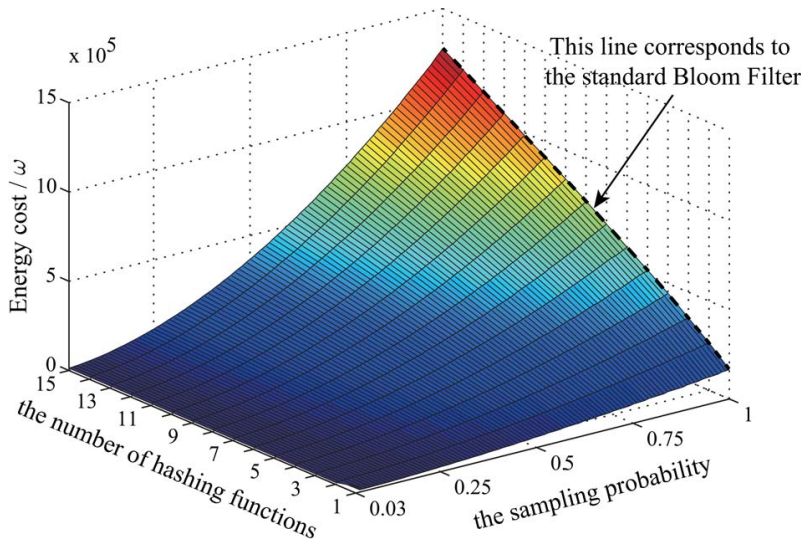

Fig. 3. Investigating the impact of $p$ and $k$ on the energy cost of SBF-UDP, where $N=10000, \lambda=100, \beta=95 \%, p$ varies from $3 \%$ to $100 \%$, and $k$ changes from 1 to 15 .

and $k$. Specifically, for fixed values of $p$ and $k$, we calculate the corresponding time cost and energy cost from steps 9 to 12. During steps $13 \sim 17$, we update the $T_{\min }$ and record the corresponding $p_{t}$ and $k_{t}$. During steps $18 \sim 22$, we update the $E_{\text {min }}$ and record the corresponding $p_{e}$ and $k_{e}$.

Algorithm 1 Find the optimal sampling probability $p$ and hashing count $k$ of SBF-UDP.

Input: The number $N$ of known tags; tolerance number $\lambda$; the reliability $\beta$.

Output: The optimal $\left\langle p_{t}, k_{t}\right\rangle$ that minimizes the time cost; The optimal $\left\langle p_{e}, k_{e}\right\rangle$ that minimizes the energy cost.

$$
\begin{aligned}
& 1: T_{\text {min }}=E_{\text {min }}=+\infty \text {; } \\
& \text { 2: } k_{t}=k_{e}=-1 \text {; } \\
& \text { 3: } p_{t}=p_{e}=-1 \text {; } \\
& \text { 4: } \delta=0.001 \text {; } \\
& \text { 5: for each } k \in[1,15] \text { do } \\
& \text { 6: } p_{\text {min }}=1-(1-\beta)^{\frac{1}{\lambda k}} \text {; } \\
& \text { 7: } p=1 \text {; } \\
& \text { 8: } \quad \text { while } p>p_{\min } \text { do } \\
& \text { 9: } \quad f=-N k p /\left\{\ln \left[\frac{1-(1-\beta)^{\frac{1}{\lambda k}}}{p}\right]\right\} \text {; } \\
& \text { 10: } \quad S=\left\lceil\frac{f}{96}\right\rceil \text {; } \\
& \text { 11: } \quad T=t_{\text {tag }}+S t_{\text {tag }}+t_{\text {short }} \text {; } \\
& \text { 12: } E=N \times\left[1+S-\sum_{i=0}^{S-1}\left(1-p+\frac{i p}{S}\right)^{k}\right] \times \omega \text {; } \\
& \text { 13: } \quad \text { if } T<T_{\min } \text { then } \\
& \text { 14: } \quad T_{\min }=T \\
& \text { 15: } \quad k_{t}=k \\
& \text { 16: } \quad p_{t}=p \\
& \text { 17: } \quad \text { end if } \\
& \text { 18: } \quad \text { if } E<E_{\min } \\
& \text { 19: } \quad E_{\min }=E \text {; } \\
& \text { 20: } \quad k_{e}=k \text {; } \\
& \text { 21: } \quad p_{e}=p \text {; } \\
& \text { 22: end if } \\
& \text { 23: end while } \\
& \text { 24: end for } \\
& \text { 25: return }\left\langle p_{t}, k_{t}\right\rangle \text { and }\left\langle p_{e}, k_{e}\right\rangle \text {; }
\end{aligned}
$$


The computational complexity of Algorithm 1 is $O\left(\frac{1}{\delta}\right)$. The computer does not need to frequently run Algorithm 1 (offline) because the parameters $N, \lambda$, and $\beta$ are not changed often in practice for monitoring applications.

5) Joint Optimization of Time and Energy Costs: Joint consideration of the time-efficiency and energy-efficiency is justified when the battery-powered active tags are used. For example, we desire to find optimal $\langle k, p\rangle$ to minimize energy while keeping the detection time within a given upper threshold. The joint optimization algorithm can be designed by slightly modifying Algorithm 1. Specifically, we only need to replace the condition at line 13 in Algorithm 1 by a new condition "if $T<T_{\min }$ and $E<E_{\text {threshold }}$ then," where $E_{\text {threshold }}$ is the maximum energy cost that is allowed. Similarly, we also replace the condition at line 18 in Algorithm 1 by a new condition "if $E<E_{\text {min }}$ and $T<T_{\text {threshold }}$ then," where $T_{\text {threshold }}$ is the maximum time cost that is allowed.

\section{Performance Evaluation}

Extensive simulation experiments are conducted to evaluate the performance of the proposed SBF-UDP in this section. We simulated the experimental conditions following related literature [18], [19], [28], [30]: (1) considering a single reader in the simulations and assuming it has adequate power to interrogate with all RFID tags; (2) the signal interference between the adjacent RFID tags is ignored. In the following subsections, we first consider an error-free communication channel between the reader and tags. Numerical results are provided to show the advantages of our sampling bloom filter over standard bloom filter. Then, we conduct simulations to compare the proposed SBF-UDP with prior schemes [18], [19], [28], [30] in terms of time-efficiency and energy-efficiency. Note that, because the identification-based protocols are far from efficient, we do not compare the proposed SBF-UDP with them. After that, experiments are conducted to show that the proposed SBFUDP indeed achieves the required detection accuracy. Finally, we conduct simulations taking the non-perfect communication channel into consideration and evaluate the impact of channel error on our SBF-UTD protocol. A simple but effective countermeasure is proposed to mitigate the negative impact of channel error, and its effectiveness is also evaluated via simulations.

\section{A. Demonstrating the Advantages of Sampling Bloom Filter}

In this set of simulations, we provide numerical results to show the advantages of our sampling bloom filter over conventional standard bloom filter. As illustrated in Fig. 4, we vary the sampling probability $p$ from $p_{\min }$ to 1 . For the purpose of clarity, we only vary the hash number $k$ from 1 to 4. The numerical results in Fig. 4 reveal that both time cost and energy cost are convex function with respect to $p$. The time (or energy) cost of traditional standard bloom filter corresponds to $p=1$. A too small sampling probability $p$ will lose too much information, and thus consuming more time and energy. A proper setting of $p$ will make our sampling bloom filter perform much better than the standard bloom filter.

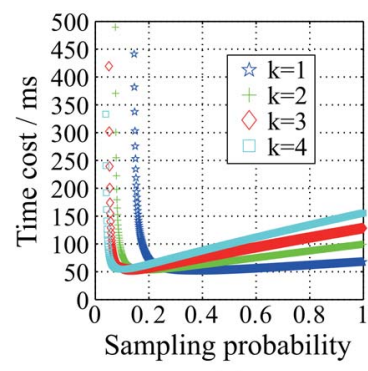

(a)

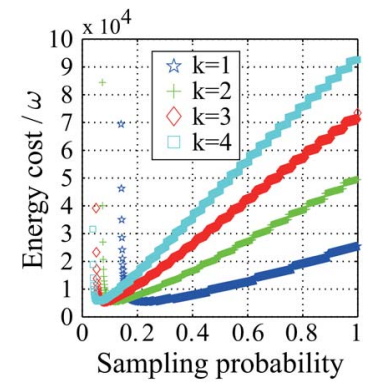

(b)
Fig. 4. Impact of $p$ on SBF-UTD. $N=3000, \lambda=20, \beta=95 \% . k$ is the number of hashing functions. (a) Time cost vs. $p$. (b) Energy cost vs. $p$.

\section{B. Comparing With the Prior Related Protocols}

Besides SEBA [18] and SEBA+ [19], we also compare our detection protocol with two representative identification protocols, i.e., CU [28] and BUIP-CF [30], which aim at pinpointing the exact unknown tags. For fair comparison, we do not simulate the process of unknown tag collection of these two identification protocols.

1) Execution Time: Experimental results shown in Table II demonstrate that the proposed SBF-UDP considerably outperforms all the previous related protocols. For example, when $N=30000, \lambda=3$, and $\beta=99 \%$, the execution time of CU, BUIP-CF, SEBA, and SEBA+ is $121.0 \mathrm{~s}, 76.6 \mathrm{~s}, 49.5 \mathrm{~s}$, and $38.5 \mathrm{~s}$, respectively. And the execution time of SBF-UDP working in time-saving mode is $4.0 \mathrm{~s}$, which represents 30 times faster than CU, 19 times faster than BUIP-CF, 12 times faster than SEBA, and 9 times faster than SEBA+.

2) Energy Cost: The simulation results shown in Table III reveal that the proposed SBF-UDP significantly reduces the energy consumption compared with the previous protocols. For example, when $N=30000, \lambda=3$, and $\beta=99 \%$, the energy cost of CU, BUIP-CF, SEBA, and SEBA+ is $4.52 \times 10^{8} \omega$, $1.13 \times 10^{8} \omega, 1.86 \times 10^{8} \omega$, and $1.92 \times 10^{8} \omega$, respectively. While the energy cost of SBF-UDP working in energy-saving mode is $1.94 \times 10^{7} \omega$, reducing the energy consumption by $95.7 \%, 82.8 \%, 89.6 \%$, and $89.9 \%$, respectively. The lifetime of active RFID tags could be significantly prolonged.

\section{The Actual Detection Reliability}

In this subsection, we conduct simulations to evaluate the actual detection reliability of the proposed SBF-UDP. The number $N$ of the known tags varies from 1000 to 10000 . The tolerance number $\lambda$ and the detection reliability $\beta$ is set to 10 and $95 \%$, respectively. We simulated different numbers $M$ of unknown tags hiding in the system, where $M$ varies from 10 to 13. The simulation results shown in Fig. 5 demonstrate that the proposed SBF-UDP protocol can meet the predefined detection reliability under both time-saving mode and energy-saving mode. Specifically, the actual detection reliability fluctuates around the predefined reliability $95 \%$ when exactly $\lambda=10$ unknown tags are simulated in Fig. 5(a), which is the most difficult case for the detection. This fluctuation because of the probabilistic variance is reasonable and acceptable in practice. 
TABLE II

Time Cost of the Protocols, Where $N$ Varies From 10000 to $30000 ; \lambda$ And $\beta$ Are Fixed to 3 And 99\%

\begin{tabular}{|c|c|c|c|c|c|c|}
\hline Alg. Name & CU & BUIP-CF & SEBA & SEBA+ & SBF-UDP(t) & SBF-UDP(e) \\
\hline $\mathrm{N}=10,000$ & $40.4 \mathrm{~s}$ & $25.6 \mathrm{~s}$ & $16.5 \mathrm{~s}$ & $12.8 \mathrm{~s}$ & $1.3 \mathrm{~s}$ & $1.7 \mathrm{~s}$ \\
\hline $\mathrm{N}=15,000$ & $60.5 \mathrm{~s}$ & $37.9 \mathrm{~s}$ & $24.7 \mathrm{~s}$ & $19.2 \mathrm{~s}$ & $2.0 \mathrm{~s}$ & $2.6 \mathrm{~s}$ \\
\hline $\mathrm{N}=20,000$ & $80 . \mathrm{s} 7$ & $51.4 \mathrm{~s}$ & $32.9 \mathrm{~s}$ & $25.6 \mathrm{~s}$ & $2.7 \mathrm{~s}$ & $3.4 \mathrm{~s}$ \\
\hline $\mathrm{N}=25,000$ & $101.3 \mathrm{~s}$ & 64.0 & $41.2 \mathrm{~s}$ & $32.0 \mathrm{~s}$ & $3.3 \mathrm{~s}$ & $4.3 \mathrm{~s}$ \\
\hline $\mathrm{N}=30,000$ & $121.0 \mathrm{~s}$ & $76.6 \mathrm{~s}$ & $49.5 \mathrm{~s}$ & $38.5 \mathrm{~s}$ & $4.0 \mathrm{~s}$ & $5.2 \mathrm{~s}$ \\
\hline
\end{tabular}

TABLE III

Energy Cost of the Protocols, Where $N$ Varies From 10000 to $30000 ; \lambda$ ANd $\beta$ Are FiXed to 3 and 99\%

\begin{tabular}{|c|c|c|c|c|c|c|}
\hline Alg. Name & CU & BUIP-CF & SEBA & SEBA+ & SBF-UDP(t) & SBF-UDP(e) \\
\hline $\mathrm{N}=10,000$ & $5.10 \times 10^{7} \omega$ & $1.25 \times 10^{7} \omega$ & $2.06 \times 10^{7} \omega$ & $2.14 \times 10^{7} \omega$ & $2.23 \times 10^{6} \omega$ & $2.17 \times 10^{6} \omega$ \\
\hline $\mathrm{N}=15,000$ & $1.13 \times 10^{8} \omega$ & $2.85 \times 10^{7} \omega$ & $4.64 \times 10^{7} \omega$ & $4.80 \times 10^{7} \omega$ & $5.00 \times 10^{6} \omega$ & $4.85 \times 10^{6} \omega$ \\
\hline $\mathrm{N}=20,000$ & $2.02 \times 10^{8} \omega$ & $5.05 \times 10^{7} \omega$ & $8.24 \times 10^{7} \omega$ & $8.55 \times 10^{7} \omega$ & $8.92 \times 10^{6} \omega$ & $8.62 \times 10^{6} \omega$ \\
\hline $\mathrm{N}=25,000$ & $3.15 \times 10^{8} \omega$ & $7.85 \times 10^{7} \omega$ & $1.29 \times 10^{8} \omega$ & $1.34 \times 10^{8} \omega$ & $1.39 \times 10^{7} \omega$ & $1.35 \times 10^{7} \omega$ \\
\hline $\mathrm{N}=30,000$ & $4.52 \times 10^{8} \omega$ & $1.13 \times 10^{8} \omega$ & $1.86 \times 10^{8} \omega$ & $1.92 \times 10^{8} \omega$ & $2.00 \times 10^{7} \omega$ & $1.94 \times 10^{7} \omega$ \\
\hline
\end{tabular}

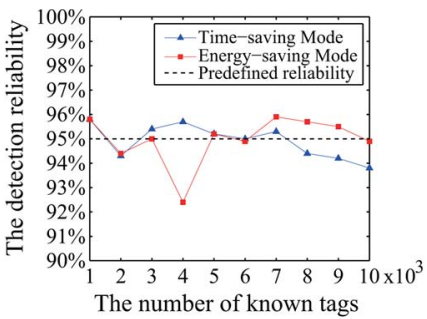

(a)

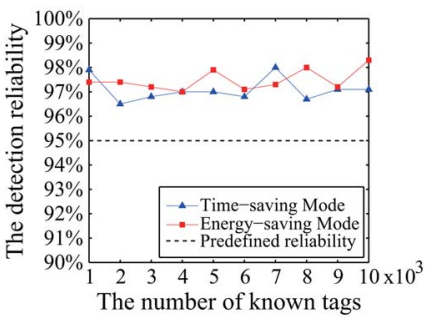

(c)

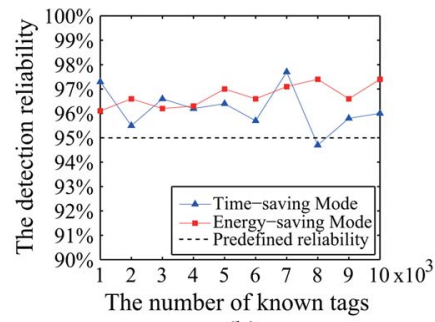

(b)

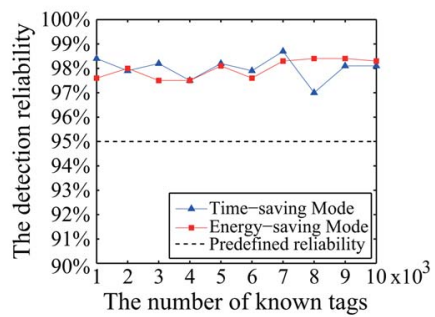

(d)
Fig. 5. Evaluating the actual detection reliability of the proposed SBF-UDP, where $N$ varies from 1000 to $10000 ; \lambda=10 ; \beta=95 \%$. (a) $\lambda=10$ unknown tag is simulated. (b) $\lambda+1=11$ unknown tags are simulated. (c) $\lambda+2=12$ unknown tags are simulated. (d) $\lambda+3=13$ unknown tags are simulated.

When more than $\lambda$ tags are simulated hiding in the system, e.g., Fig. 5(b)-(d), the actual detection reliability is usually larger than the predefined reliability $95 \%$.

\section{The Impact of Channel Error}

For the clarity of description, this paper assumed (so far) a perfect communication channel between the reader and tags [10], [24]. We now propose a countermeasure to mitigate negative impact of channel error on our SBF-UTD protocol. The $f$-bit bloom filter is divided into multiple segments, each of which is 80-bit. The computer calculates CRC-16 (cyclic redundancy check) of each segment. The reader sequentially broadcasts the binary $\langle$ segment, $C R C\rangle$ to the tags. The 96-bit binary can be transmitted by the reader in a tag slot. We assume each bit in the binary is corrupted due to channel error with a probability of $P_{B E R}$ during the transmission process. If some bits in the binary $\langle$ segment, $C R C\rangle$ are corrupted, the segment and the CRC confined in a binary will not match. If a tag finds the received $\langle$ segment, $C R C\rangle$ is corrupted, it will

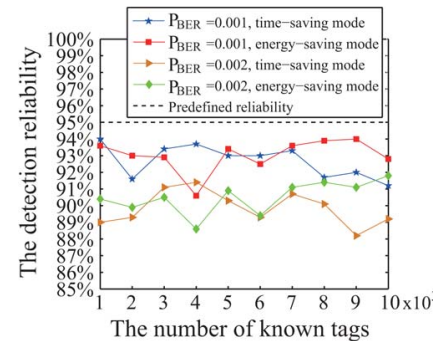

(a)

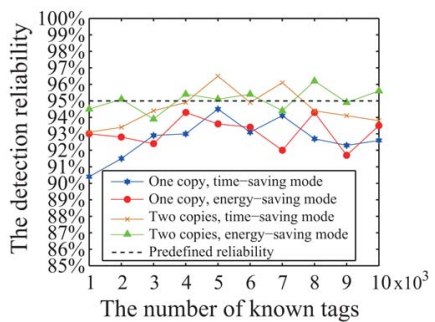

(b)
Fig. 6. Evaluating the impact of channel error on the accuracy of SBF-UTD, where $N$ varies from 1000 to $10000 . \lambda=10, \beta=95 \% .10$ unknown tags are simulated. (a) Investigating the impact of different $P_{B E R}$. (b) Investigating the effectiveness of multi-copy countermeasure.

not use the segment to check whether it is unknown tag. A simple but effective countermeasure to mitigate the impact of channel error is to broadcast $c>1$ copies of $\langle$ segment, $C R C\rangle$. Multiple copies can increase the probability that at least one of them is correctly transmitted. With fixed $N, \lambda$ and $\beta$, the time and energy costs will be proportional to the number of copies transmitted. The simulation results in Fig. 6(a) show that the channel error decreases the detection accuracy, and the results in Fig. 6(b) demonstrate that our multi-copy countermeasure is able to mitigate the negative impact of channel error.

\section{CONCLUSION}

This study has investigated a practically important problem of unknown tag detection that aims to detect the existence of unknown tags in a time- and energy-efficient way. We have proposed a new Sampling Bloom Filter which is a general case of the standard Bloom Filter. Based on the new filtering technique, we have further proposed the Sampling Bloom Filter based Unknown tag Detection Protocol (SBF-UDP). Sufficient theoretically analysis is presented to minimize the execution time as well as energy consumption of the proposed protocol. We conduct extensive simulations to evaluate the performance of our SBF-UDP, and the experimental results show that our SBF-UDP protocol considerably outperforms the previous related protocols in terms of both time-efficiency and energy-efficient. 


\section{REFERENCES}

[1] L. Yang et al., "Tagoram: real-time tracking of mobile RFID tags to high precision using COTS devices," in Proc. ACM MobiCom, 2014, pp. 237248.

[2] A. Matic, A. Papliatseyeu, V. Osmani, and O. Mayora-Ibarra, "Tuning to your position: FM radio based indoor localization with spontaneous recalibration," in Proc. IEEE PerCom, 2010, pp. 153-161.

[3] W. Zhu, J. Cao, Y. Xu, L. Yang, and J. Kong, "Fault-tolerant RFID reader localization based on passive RFID tags," in Proc. IEEE INFOCOM, 2012, pp. 2183-2191.

[4] L. Xie, H. Han, Q. Li, J. Wu, and S. Lu, "Efficiently collecting histograms over RFID tags," in Proc. IEEE INFOCOM, 2014, pp. 145-153.

[5] M. Chen, W. Luo, Z. Mo, S. Chen, and Y. Fang, "An efficient tag search protocol in large-scale," in Proc. IEEE INFOCOM, 2013, pp. 899-907.

[6] K. Bu, B. Xiao, Q. Xiao, and S. Chen, "Efficient misplaced-tag pinpointing in large RFID systems," IEEE Trans. Parallel Distrib. Syst., vol. 23, no. 11, pp. 2094-2106, Nov. 2012.

[7] S. Qi, Y. Zheng, M. Li, L. Lu, and Y. Liu, "COLLECTOR: A secure RFID-enabled batch recall protocol," in Proc. IEEE INFOCOM, 2014, pp. 1510-1518.

[8] Y. Zheng and M. Li, "Fast tag searching protocol for large-scale RFID," in Proc. IEEE ICNP, 2011, pp. 363-372.

[9] H. Liu et al., "Generic composite counting in RFID systems," in Proc. IEEE ICDCS, 2014, pp. 597-606.

[10] B. Sheng, C. C. Tan, Q. Li, and W. Mao, "Finding popular categories for RFID tags," in Proc. ACM MobiHoc, 2008, pp. 1-10.

[11] M. Shahzad and A. X. Liu, "Every bit counts: Fast and scalable RFID estimation," in Proc. ACM MobiCom, 2012, pp. 365-376.

[12] M. Shahzad and A. X. Liu, "Probabilistic optimal tree hopping for RFID identification," in Proc. ACM SIGMETRICS, 2013, pp. 293-304.

[13] F. C. Schoute, "Dynamic frame length ALOHA," IEEE Trans. Commun., vol. 31, no. 4, pp. 565-568, Apr. 1983.

[14] P. Popovski, K. Fyhn, R. M. Jacobsen, and T. Larsen, "Robust statistical methods for detection of missing RFID tags," IEEE Wireless Commun., vol. 18, no. 4, pp. 74-80, Aug. 2011.

[15] W. Bishop, "Documenting the value of merchandising," Nat. Assoc. Retail Merchandising Service, Plover, WI, USA, 2003.

[16] Y. Yin, L. Xie, S. Lu, and D. Chen, "Efficient protocols for rule checking in RFID systems," in Proc. IEEE ICCCN, 2013, pp. 1-7.

[17] W. Luo, S. Chen, T. Li, and Y. Qiao, "Probabilistic missing-tag detection and energy-time tradeoff in large-scale RFID systems," in Proc. ACM MobiHoc, 2012, pp. 95-104.

[18] L. Yang, J. Han, Y. Qi, and Y. Liu, "Identification-free batch authentication for RFID tags," in Proc. IEEE ICNP, 2010, pp. 154-163.

[19] G. Bianchi, "Revisiting an RFID identification-free batch authentication approach," IEEE Commun. Lett., vol. 15, no. 6, pp. 632-634, Jun. 2011

[20] P. Semiconductors, I-CODE Smart Label RFID Tags, Jan. 2004. [Online]. Available: http://www.nxp.com/acrobat_download/other/identification/ SL092030.pdf

[21] C. C. Tan, B. Sheng, and Q. Li, "Efficient techniques for monitoring missing RFID tags," IEEE Trans. Wireless Commun., vol. 9, no. 6, pp. 18821889, Jun. 2010

[22] W. Luo, S. Chen, T. Li, and S. Chen, "Efficient missing tag detection in RFID systems," in Proc. IEEE INFOCOM, 2011, pp. 356-360.

[23] T. Li, S. Chen, and Y. Ling, "Identifying the missing tags in a large RFID system," in Proc. ACM MobiHoc, 2010, pp. 1-10.

[24] R. Zhang, Y. Liu, Y. Zhang, and J. Sun, "Fast identification of the missing tags in a large RFID system," in Proc. IEEE SECON, 2011, pp. 278-286.

[25] X. Liu, K. Li, G. Min, Y. Shen, A. X. Liu, and W. Qu, "Completely pinpointing the missing RFID tags in a time-efficient way," IEEE Trans. Comput., vol. 64, no. 1, pp. 87-96, Jan. 2015.

[26] Y. Zheng and M. Li, "P-MTI: Physical-layer missing tag identification via compressive sensing," in Proc. IEEE INFOCOM, 2013, pp. 917-925.

[27] X. Liu et al., "Efficient unknown tag identification protocols in largescale RFID systems," IEEE Trans. Parallel Distrib. Syst., vol. 25, no. 12, pp. 3145-3155, Dec. 2014.

[28] B. Sheng, Q. Li, and W. Mao, "Efficient continuous scanning in RFID systems," in Proc. IEEE INFOCOM, 2010, pp. 1-9.

[29] S. Lee, S. Joo, and C. Lee, "An enhanced dynamic framed slotted ALOHA algorithm for RFID tag identification," in Proc. IEEE MobiQuitous, 2005, pp. 166-172.

[30] X. Liu, S. Zhang, K. Bu, and B. Xiao, "Complete and fast unknown tag identification in large RFID systems," in Proc. IEEE MASS, 2012, pp. $47-55$

[31] X. Liu et al., "A fast approach to unknown tag identification in large scale RFID systems," in Proc. IEEE ICCCN, 2013, pp. 1-7.
[32] B. Bloom, "Space/time tradeoffs in hash coding with allowable errors," ACM Commun., vol. 13, no. 7, pp. 422-426, Jul. 1970.

[33] H. Fang, K. Murali, and L. TV, "Building high accuracy bloom filters using partitioned hashing," ACM SIGMETRICS Perform. Eval. Rev., vol. 35 , no. 1, pp. 277-288, Jun. 2007.

[34] K. Huang et al., "A multi-partitioning approach to building fast and accurate counting bloom filters," in Proc. IEEE IPDPS, 2013, pp. 1159-1170.

[35] I. Chlamtac, C. Petrioli, and J. Redi, "Energy-conserving access protocols for identification networks," IEEE/ACM Trans. Netw., vol. 7, no. 1, pp. 51-59, Feb. 1999.

[36] X. Liu et al., "Efficient detection of cloned attacks for large-scale RFID systems," in Proc. ICA3PP, 2014, pp. 85-99.

[37] Y. Qiao, T. Li, and S. Chen, "One memory access bloom filters and their generalization," in Proc. IEEE INFOCOM, 2011, pp. 1745-1753.

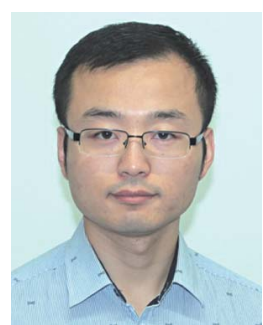

Xiulong Liu received the B.E. degree from the School of Software Technology, Dalian University of Technology, China, in 2010. Currently, he is pursuing the Ph.D. degree in the School of Computer Science and Technology, Dalian University of Technology, China. His research interests include RFID systems and wireless sensor networks.

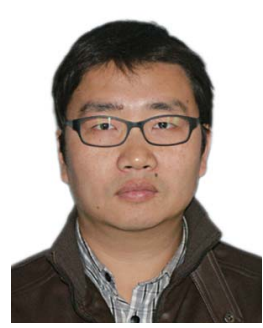

Heng Qi received the bachelor's degree from Hunan University in 2004 and the the master's and doctoral degree from Dalian University of Technology in 2006 and 2012, respectively. He is a Lecturer at the School of Computer Science and Technology, Dalian University of Technology, China. His research interests include computer network, wireless network and multimedia computing.

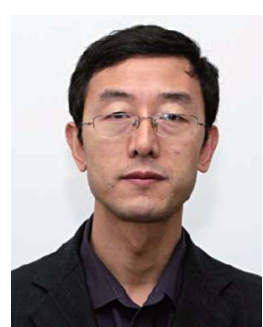

Keqiu Li (SM'14) received the bachelor's and master's degrees from the Department of Applied Mathematics at the Dalian University of Technology in 1994 and 1997, respectively. He received the Ph.D. degree from the Graduate School of Information Science, Japan Advanced Institute of Science and Technology in 2005. He also has two-year postdoctoral experience at the University of Tokyo, Japan. $\mathrm{He}$ is currently a Professor in the School of Computer Science and Technology, Dalian University of Technology, China. He has published more than 100 technical papers in such journals as IEEE TRANSACTIONS ON PARALLEL AND DISTRIBUTED SYSTEMS (TPDS), ACM Transactions on Internet Technology (TOIT). and ACM Transactions on Multimedia Computing, Communications, and Applications, $\mathrm{He}$ is an Associate Editor of IEEE TPDS and EEE TRANSACTIONS ON COMPUTERS (TC). His research interests include Internet technology, data center networks, cloud computing, and wireless networks.

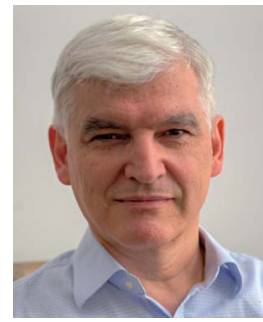

Ivan Stojmenovic (F'08) was Editor-in-Chief of IEEE TRANSACTIONS ON PARALLEL AND DISTRIBUTED SYSTEMS (2010-2013), and was founder of three journals. He was Editor of IEEE TRANSACTIONS ON COMPUTERS, IEEE NETWORK, IEEE TRANSACTIONS ON ClOUd COMPUTING, and ACM Wireless Networks and a member of the steering committee member of IEEE TRANSACTIONS ON EMERGENT TOPICS IN COMPUTING. He is on Thomson Reuters list of Highly Cited Researchers from 2013, has top h-index in Canada for mathematics and statistics, and has more than 15000 citations. He has received several Best Paper Awards. He is Fellow of Canadian Academy of Engineering and Academia Europaea. He received Humboldt Research Award. 


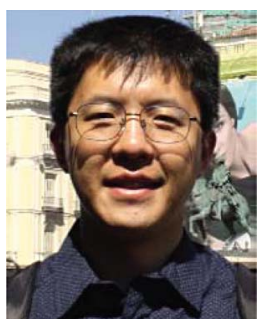

Alex X. Liu received the Ph.D. degree in computer science from the University of Texas at Austin in 2006. $\mathrm{He}$ is an Associate Professor with the Department of Computer Science and Engineering, Michigan State University. He is an Associate Editor of IEEE/ACM TRANSACTIONS ON NETWORKING and an Area Editor of Journal of Computer Communications (Elsevier). He received the IEEE \& IFIP William C. Carter Award in 2004 and an NSF CAREER award in 2009. He received the Withrow Distinguished Scholar Award in 2011 at Michigan State University. He received Best Paper Awards from ICNP-2012, SRDS2012, and LISA-2010. His research interests focus on networking and security.

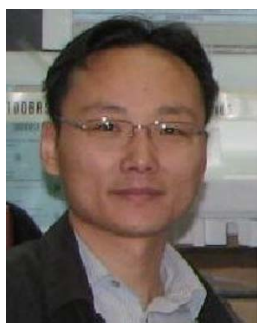

Yanming Shen received the B.S degree in automation from Tsinghua University, Beijing, China, in 2000, and the Ph.D. degree from the Department of Electrical and Computer Engineering at the Polytechnic Institute of New York University, Brooklyn, in 2007. He is a Professor with the School of Computer Science and Technology, Dalian University of Technology, DaLian, China. His general research interests include packet switch design, data center networks, peer-to-peer video streaming, and algorithm design, analysis, and optimization. He is a recipient of the 2011 Best Paper Awards for Multimedia Communications (awarded by IEEE Communications Society).

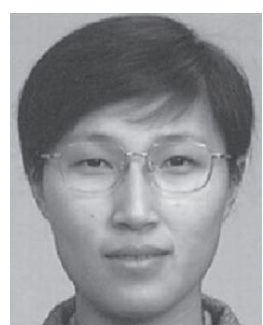

Wenyu Qu received the bachelor's and master's degrees from Dalian University of Technology, China in 1994 and 1997, and the doctorate degree from Japan Advanced Institute of Science and Technology in 2006. She is a Professor at the School of Information and Technology, Dalian Maritime University, China. She was a Lecturer in Dalian University of Technology from 1997 to 2003 . Her research interests include mobile agent-based technology, distributed computing, computer networks, and grid computing. She has published more than 50 technical papers in international journals and conferences. She is on the committee board for a couple of international conferences.

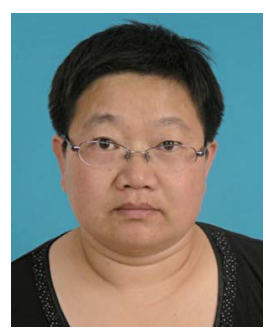

Weilian Xue received the bachelor's and master's degrees both from Liaoning Normal University, China in 1989 and 2003, and the doctoral degree from Dalian University of Technology, China in 2011. She is a Professor at the School of Management, Liaoning Normal University. Her research interests are in wireless networks, cloud computing, data mining, and information systems. 